\title{
Targeting the neural task-control circuitry to enhance self-regulatory control in bulimia nervosa
}

\section{By Dr Jessica K. Edwards}

Previous research has found that bulimia nervosa is associated with dysregulated self-regulatory control, as a result of anatomical and functional disturbances to the neural task-control circuitry in the brain ${ }^{1}$. A longitudinal study has now assessed how activation of these taskcontrol circuits varies with changes in bulimia nervosa symptoms that occur during adolescence. Participants $(32$ females with bulimia nervosa and 28 healthy controls) were invited to complete a Simon task that involved presentation of a leftward or rightward pointing arrow that was either congruent or incongruent with its position on a screen. The participants were asked to report the direction of the arrow as quickly as possible, whilst the neural task-control circuits were monitored by functional magnetic resonance imaging. The procedure was repeated at three time points over adolescence. Healthy controls showed decreased conflictrelated neural activation over time, whereas those with bulimia nervosa showed increased activation of the taskcontrol circuits. Interestingly, bulimia symptoms inversely predicted conflict-related neural activation over time, meaning that as symptoms decreased, activation of the task-control circuitry increased. The researchers propose that this phenomenon may be a compensatory mechanism that permits regulation of eating behaviours over childhood development. They conclude that the neural task-control circuitry may be an anatomical target for early intervention in bulimia nervosa to enhance self-regulatory control.
Cyr, M., Fontaine, M., Stefan, M., Terranova, K., Kopala-Sibley, D.C., Attia, E. \& Marsh, R. (2017), A longitudinal functional magnetic resonance imaging study of task control circuits and bulimic symptoms over adolescence. J Child Psychol Psychiatr. doi:10.1111/jcpp.12840

\section{Further reading:}

Lock, Garrett, Beenhakker, \& Reiss, 2011; Marsh et al., 2011) and adults (Marsh et al., 2009; Skunde et al., 2016

'Lock, J., Garrett, A., Beenhakker, J \& Reiss, A.L. (2011), Aberrant brain activation during a response inhibition task in adolescent eating disorder subtypes. Am J Psychiatry. 168:55-64. doi: 10.1176/appi.ajp.2010.10010056

\section{Glossary:}

Bulimia nervosa: an eating disorder characterized by recurrent episodes of binge eating followed by compensatory behaviours to prevent weight gain

Self-regulatory control: conscious management of guiding ones thoughts, behaviours and desires to reach goals; it encompasses executive control, emotional regulation and the ability to delay gratification

Simon task: a paradigm described by J.R. Simon in 1963 whereby people respond faster and more accurately if there is a match between a stimulus and its response feature (the "stimulus-response compatibility effect")

Functional magnetic resonance imaging: a non-invasive technique that uses a strong, static magnetic field and radio waves to measure brain activity based on detecting changes in cerebral blood flow 